\title{
Quantum phase transitions of strongly correlated metals
}

\section{Transiciones de fase cuántica de materiales fuertemente correlacionados}

Martha Y. Suárez Villagrán'

Nikolaos Mitsakos ${ }^{2}$ and John H. Miller Jr'

DOI: 10.29151/reit.n1a1

${ }^{1}$ Department of Physics \& Texas Center for Superconductivity, University of Houston, Houston, Tx 77204-5005 USA

${ }^{2}$ Department of Mathematics, University of Houston, Houston, Tx 77204-5008 USA 

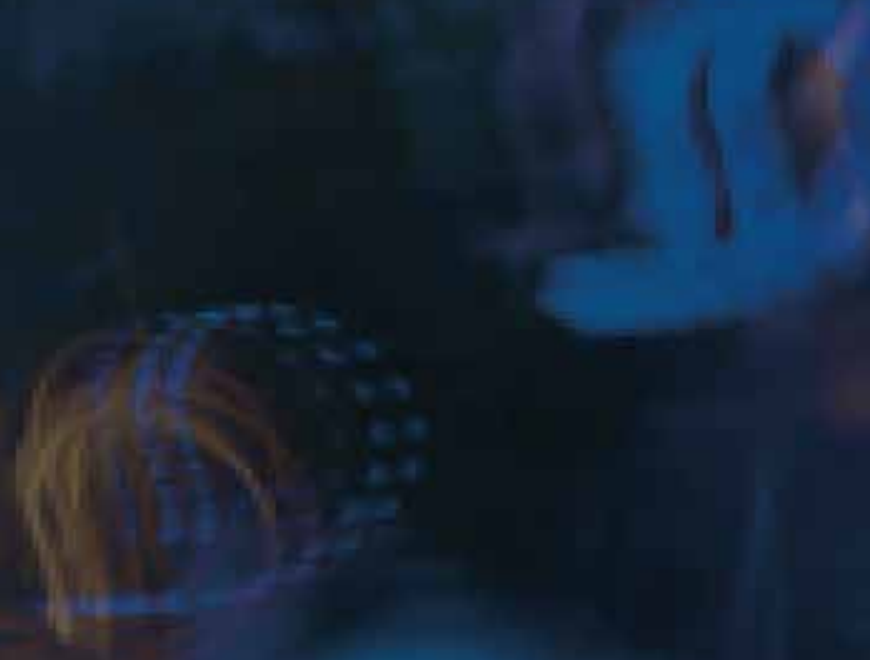


\title{
Resumen
}

En este artículo, discutimos varios aspectos de la transición de fase cuántica, con especial énfasis en la transición metal-aislante. Comenzamos con una revisión de trabajos claves experimentales y teóricos realizado por científicos que hicieron contribuciones históricamente importantes al campo. Posteriormente discutimos cómo la adición de dopaje a un sistema reduce la temperatura crítica de la transición de fase. Aunque muchos aspectos de la transición de fase cuántica siguen siendo un problema abierto, se han hecho progresos considerables para revelar la física subyacente, tanto teórica como experimentalmente.

Palabras clave: Transición de fase cuántica; transición metal aislante; DMFT; StatDMFT; localización de Anderson.

\begin{abstract}
In this article, we discuss several aspects of the quantum phase transition, with special emphasis on the metalinsulator transition. We start with a review of key experimental and theoretical works and then discuss how doping a system reduces the critical temperature of the overall phase transition. Although many aspects of the quantum phase transition still remain an open problem, considerable progress has been made in revealing the underlying physics, both theoretically and experimentally.

Keywords: quantum phase transition; metal insulator transition; DMFT; StatDMFT; Anderson localization.
\end{abstract}




\section{Introduction and discussion}

The flow of electrons in a metal was first successfully described in a model by Paul Drude in 1900 [1]. This semi-classical theory assumes that electrons move freely through crystal without feeling the effects of other electrons, but can be scattered by crystalline ions, where the average time interval between scattering events is known as the relaxation time. This simple model enables description of a constant current due to an applied electric field [1]. The Drude theory was modified by Arnold Sommerfeld in 1927. His free electron model includes quantum effects, such as the Fermi-Dirac distribution and Pauli exclusion principle. The theoretical basis for understanding why this model describes the behavior of various materials was established by Lev Landau with his Fermi liquid theory [2]. This theory maps elementary excitations of interacting electronic systems onto excitations of non-interacting systems, describing weak residual interactions with a small set of phenomenological parameters. The primary result of Fermi liquid theory is that low energy excitations, known as quasiparticles, behave almost like non-interacting electrons. The relaxation time of a quasiparticle state diverges as its energy approaches the Fermi energy, $E F$, and as $T \rightarrow 0$.

In highly correlated electronic systems, the effects of interactions between electrons cannot be neglected. Such effects lead to interesting phenomena, including the metal-insulator phase transition, which have attracted considerable attention during the last several years. Phase transitions are fascinating phenomena observed in a wide variety of chemical, physical, and biological systems. They encompass a range of phenomena that in- clude: (i) transitions between solid, liquid, and gaseous phases of matter; (ii) transitions between paramagnetism and several magnetically ordered phases (ferromagnetic, antiferromagnetic, and ferrimagnetic); (iii) the superconducting transition; (iv) the superfluid transition; and many others. One characteristic of a phase transition is a singular change in various physical properties of the system. Phase transition is accompanied by the appearance of some sort of spontaneous ordering or symmetry breaking. Examples of orders that emerge are the crystalline order of solids, the breaking of rotational symmetry and time reversal symmetry in magnetic systems, and the "off-diagonal long-range order" present in superconductors and superfluids.

Phase transitions can occur even at zero temperature, with variation of some state parameters, such as pressure, chemical pressure, magnetic field, or electric field. A phase transition at $\mathrm{T}=0$ is called a quantum phase transition. It is characterized by singular change in the fundamental state of the system. It is important to note that although zero temperature is impossible to achieve, the effects of a quantum phase transition at $\mathrm{T}=0$ can still be felt at finite temperatures [3]. It is thus important to study quantum phase transitions, due to their impact on real systems and many potential applications.

Understanding systems' behavior close to phase transition, induced by electron-electron interaction, remains at the epicenter of scientific and technological research. One ingredient is the distinction between metallic and insulating behavior, which is only well defined at zero temperature. As $T \rightarrow 0$, resistivity of an insulator diverges, while that of a metal converges. At temperatures further away from zero, resistivity is bounded in both cases. As a result, one can consider the metal-in- 
sulator transition as, essentially, a quantum phase transition. However, several systems exhibit an abrupt jump in conductivity by several orders of magnitude at finite temperatures. It is therefore natural to extend the metal-insulator transition concept to finite temperatures. The theoretical description of this extension started with Mott's work [4], and grew further with Hubbard's contribution [5], and the description of Brinkman and Rice [6]. Dynamical Mean Field Theory (DMFT) unified the views of Hubbard and Brinkman and Rice and has become the most widely accepted scheme for theoretical description of the Mott transition.

The aforementioned theoretical tools allow us to explain the behavior of compounds such as transition metal oxides. One of the first compounds where Mott transition was ever observed is $\mathrm{V}_{2} \mathrm{O}_{3}$ doped with chromium or titanium [7]. The transition had been induced by varying pressure, chemical potential, and/or temperature. Since then, the metal-insulator transition has been observed in other physical systems, including: (i) doped semiconductors (such as Si doped with P or B $[8,9]$ ); (ii) two-dimensional electron systems in MOSFETs ("metal-oxide-semiconductor field-effect transistors" [10]) or semiconductor heterostructures (GaAs / AlGaAs) [11, 12]; (iii) systems containing transition metals $\left(\mathrm{V}_{2} \mathrm{O}_{3}[13], \mathrm{VO}_{2}\right.$ [14], NiSSe [15], $\mathrm{Nb}$ [16]); and (iv) organic conductors (for example, $\kappa-(\mathrm{BETD} T \mathrm{TTF})_{2} \mathrm{Cu}\left[\mathrm{N}(\mathrm{CN})_{2}\right] \mathrm{Cl}$ [17]). The transition can now be induced by varying pressure, chemical potential, concentration of dopants, or temperature. Many of these systems are not pure, exhibiting intrinsic or extrinsic disorder.

There are several known mechanisms capable of transforming a metal into an insulator. In the absence of electron-electron interactions, a sufficiently high level of disorder leads to localization of the particle's wave function, the so-called Anderson localization [18]. Much is known about this mechanism, in particular, a successful scaling theory [19] showed that all states of a particle are localized in the presence of any level of disorder in systems of reduced dimension, $d \leq 2$.

When $d>2$, it is necessary to add a minimum amount of disorder to transform the metal into an insulator. This transition is known as the Anderson metal-insulator transition.

Mott proposed that, even in the absence of disorder, electron-electron interactions can, under certain circumstances, induce a metal-insulator transition [20]. Although Mott's original mechanism was essentially based on the long-range character of the Coulomb interactions, a model with interactions of short range was proposed by Hubbard [21, 22, 23]. In this context, a metal-insulator transition may occur for sufficiently strong electronic interactions when there is one valence electron per site of the crystalline network. Transitions induced by electron-electron interactions are known as Mott or Mott-Hubbard transitions. Understanding the interplay between disorders and interactions $[24,25,26,27]$, despite the progress achieved, remains an essentially open problem [3].

Several approaches have been introduced to theoretically describe the Mott transition with Hubbard's series of contributions [21, 22, 23] being one of the first. Starting with the so-called atomic limit, where electron-electron interaction energy is much greater than the kinetic energy of each electron, and the system behaves as an insulator, Hubbard gradually reduces the value of that interaction. The characteristic gap of the Mott insulator, which separates two excitation 
bands, known as Hubbard bands, closes at a critical value $U=U_{c H u b b}$ and the system becomes metallic. Brinkman and Rice follow an alternative path [28]. Using the variational wave function proposed by Gutzwiller [29, 30, 31], they analyze how correlated metallic behavior collapses by increased electronic interactions. In this case, at a certain critical value of the $U=U_{C B R}$ interaction, the quasiparticles of the strongly correlated Fermi liquid disappear and the system becomes insulator. While Hubbard's approach cannot effectively describe the quasiparticles of the correlated metal, the Brinkman-Rice approach does not predict the presence of Hubbard bands. Still, both features are observed experimentally, for example in optical conductivity measurements, which highlights the insufficiency of these two approaches.

\section{Results and Conclusions}

Dynamical Mean Field Theory (DMFT) [32, 33] allowed a more concise description of the Mott transition by unifying the view of Hubbard with that of Brinkman and Rice. DMFT can incorporate, for intermediate values of the interaction $U$, both the low-energy Fermi liquid particles and the Hubbard bands at high energies. In this context, the Mott transition is a first order transition, characterized by the disappearance of quasi-particles, with only the finite energy excitations of the Hubbard bands present. The transition is characterized by a region of coexistence between the metal and the insulator, as in the case of supercooling and superheating of the liquid-gas transition. The first order transition line in the diagram of temperature phase versus $U$ interaction (Fig. 1) ends at a second-order critical point $\left(T_{c}, U_{c}\right)$. Below $T_{c}$, resistivity drops abruptly as a function of $U$. This drop smoothens with increasing temperature and finally disappears at the critical point [3].

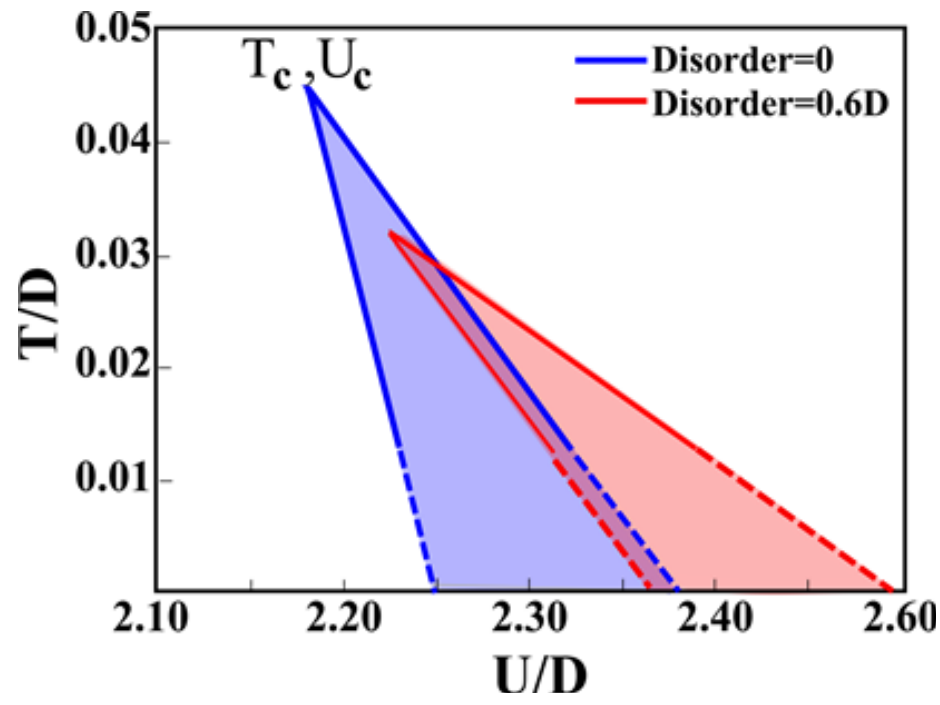

Fig 1: $T \times U$ phase diagram for the system with and without disorder

DMFT enables the study of strongly correlated electron systems and, with respect to the Mott metal-insulator transition, allows a satisfactory description in simplified models such as the Hubbard model. Its generalization, the Statistical Dynamic Mean Field Theory (StatDMFT), describes disordered systems even more adequately by assuming that various lattice sites have different energies described by a probability distribution, usually uniform or Gaussian [3].

Experimental measurements made on $\mathrm{NiS}_{2-x \mathrm{Sex}}$ and organic compounds demonstrate that the presence of disorder causes the temperature of the critical point to decrease. Experiments conducted on $\mathrm{NiS}_{2}$, for example, show that the critical point at which the Mott transition occurs is 150 $\mathrm{K}$, under external pressure of $3 \mathrm{GPa}$. On the other hand, when $\mathrm{Se}$ is substituted for $S$, yielding $\mathrm{NiS}_{2-\mathrm{SS}}$, the critical point is reduced to $100 \mathrm{~K}$ [34, 35]. 
Although many characteristics of the phase transitions still remain as an open problem, considerable progress has been made to reveal the underlying physics, both theoretically and experimentally. Even greater progress will likely be enabled by advances in the latest, most sophisticated tools, such as machine learning and deep learning [36].

\section{Acknowledgments}

M.Y.S.V and J.H.M acknowledge support by the Texas Center for Superconductivity at the University of Houston and the University of Houston Health Research Institute.

\section{References}

[1] N. D. Mermin e N. W. Aschcroft, Solid State Physics, Saunders College, (1988).

[2] L. D. Landau e E. M. Lifshitz, Statistical Physics, Addison-Wesley, Reading. MA(1969).

[3] M. Y. Suárez-Villagrán, N. Mitsakos, T. H. Lee, V. Dobrosavljevic, J. H. Miller, Jr, E. Miranda, E, Phys. Rev. B 101, 235112 (2020).

[4] N. F. Mott, The Basis Of the Electron Theory of Metals with Special Reference to the transition Metals, Proc. Phys. Soc., 416-422, 62, (1949).

[5] J. Hubbard, Electron Correlations in Narrow Energy Bands. III. An Improved Solution, Proc. Roy. Soc. (London), 401-419, 281, (1964).

[6] W. F. Brinkman, T. M. Rice, Application of Gutzwiller's Variational Method to the Metal-Insulator Transition, Phys. Rev. B, 43024304, 2, (1970).

[7] D. B. McWhan, A. Menth, J. P. Remeika, W. F. Brinkman, and T. M. Rice, Metal-Insulator Transition in Pure and Doped V2O3, Phys. Rev. B., 1920-1932, 7, (1973).

P. Limelette, A. Georges, D. J'erome, P. Wzietek, P.
Metcalf, J. M. Honig, Universality and Critical Behavior at the Mott Transition, Science, 89-92, 302, (2003).

[8] T. F. Rosenbaum, R. F. Milligan, M. A. Paalanen, G. A. Thomas, R. N. Bhatt, W. Lin. Metal-insulator transition in a doped semiconductor. Phys. Rev. B 1983, 27, 7509-7523.

[9] M. A. Paalanen, R. N. Bhatt. Transport and thermodynamic properties across the metal-insulator transition. Physica B 1991, 169, 223230. [10] S. Anissimova, S. V. Kravchenko, A. Punnoose, A.M. Finkel'stein, T.M. Klapwijk. Flow diagram of the metal insulator transition in two dimensions. Nature Phys 2007, 3, 707-710

[11] Y. Hanein, U. Meirav, D. Shahar, C.C. Li, D.C. Tsui, Hadas Shtrikman. The Metalliclike Conductivity of a Two-Dimensional Hole System. Phys. Rev. Lett. 1998, 80, 1288-1292.

[12] M. P. Lilly, J. L. Reno, J. A. Simmons, I. B. Spielman, J. P. Eisenstein, L. N. Pfeiffer, K. W. West, E. H. Hwang, S. Das Sarma. Resistivity of Dilute 2D Electrons in an Undoped GaAs Heterostructure. Phys. Rev. Lett. 2003, 90, 056806.

[14] M. M. Qazilbash, M. Brehm, a. P. Byung-Gyu Chae, G. O. Andreev, Bong-Jun Kim, Sun Jin Yun, A.V. Balatsky, M. B. Maple, F. Keilmann, Hyun-Tak Kim, D. N. Basov. Mott Transition in $\mathrm{V}$ O2 Revealed by Infrared Spectroscopy and Nanolmaging. Science 2007, 318, 1750-1753.

[15] J. Mazzaferro, H. Ceva, B. Alascio. Intermediate-valence effects on the phase diagram of N iS2 - xSex. Phys. Rev. B. 1980, 22, 353.

[16] P. Lederer, H. Launois, J. P. Pouget, A. Casalot, $\mathrm{G}$. Villeneuve. Contribution to the study of the metal-insulator transition in the $\mathrm{V} 1-\mathrm{xN}$ bxO2 system-II magnetic properties. Journal of Physics and Chemical of Solid 1972, 33, 1961-1967. 
[17] P. Limelette, P. Wzietek, S. Florens, A. Georges, T. A. Costi, C. Pasquier, D. Jerome, C. Meziere, P. Batail. Mott Transition and Transport Crossovers in the

Organic Compound k - (BEDT - T T F)2Cu[N(CN)2] Cl. Phys. Rev. Lett. 2003, 91, 016401.

[18] P. W. Anderson. Absence of Diffusion in Certain Random Lattices. Phys. Rev. 1958, 109, 492.

[19] E. Abrahams, P. W. Anderson, D. C. LicciardeIlo, T. V. Ramakrishnan. Scaling Theory of Localization: Absence of Quantum Diffusion in Two Dimensions. Phys. Rev. Lett. 1979, 42, 673.

[20] N. F. Mott, Metal-Insulator transition, Taylor \& Francis, London, 1990.

[21] J. Hubbard. Electron correlations in narrow energy bands. Proc. R. Soc. (London) A 1963, 276, 238.

[22] J. Hubbard. Electron correlations in narrow energy bands- II- The degenerate band case. Proc. Roy. Soc. (London) A 1964, 277, 237.

[23] J. Hubbard. Electron correlations in narrow energy bands - III - An improved solution. Proc. Roy. Soc. (London) A 1964, 281, 401.

[24] C. Castellani, C. D. Castro, P. A. Lee, M. Ma. Interaction-driven metal-insulator transitions in disordered fermion systems. Phys. Rev. B 1984, 30, 527.

[25] C. Castellani, B. G. Kotliar, P. A. Lee. Fermiliquid theory of interacting disordered systems and the scaling theory of the metalinsulator transition. Phys. Rev. Lett. 1987, 56, 1179.

[26] P. A. Lee, T. V. Ramakrishnan. Disordered electronic systems. Rev. Mod. Phys. 1985, 57,
287.

[27] B. L. Altshuler, A. G. Aronov. Zero bias anomaly in tunnel resistance and electron-electron interaction. Solid State Commun. 1979, 30, 115.

[28] W. F. Brinkman, T. M. Rice. Application of Gutzwiller's Variational Method to the Metal-Insulator Transition. Phys. Rev. B 1970, 2, 4302.

[29] M. C. Gutzwiller. Effect of Correlation on the Ferromagnetism of Transition Metals. Phys. Rev. Lett. 1963, 10, 159-162.

[30] M. C. Gutzwiller. Effect of Correlation on the Ferromagnetism of Transition Metals. Phys. Rev. 1964, 134, A923-A941.

[31] M. C. Gutzwiller. Correlation of Electrons in a Narrow s Band. Phys. Rev. 1965, 137, A1726A1735.

[32] W. Metzner, D. Vollhardt. Correlated Lattice Fermions in $d=\infty$ Dimensions. Phys. Rev. Lett. $1989,62,324-327$.

[33] A. Georges, G. Kotliar, W. Krauth, M. J. Rozenberg. Dynamical mean-field theory of strongly correlated fermion systems and the limit of infinite dimensions. Rev.

Mod. Phys. 1996, 68, 13.

[34] Y. Sekine, H. Takahashi, N. Mori, T. Matsumoto, and T. Kosaka, Physica B 237, 148 (1997).

[35] S. Miyasaka, H. Hiraka, K. Yamada, and Y. Endoh, J. Phys. Soc. Jpn 69, 1503 (2000).

[36] Villagrán, M., Mitsakos, N., Miller, J. \& Azevedo, R. Using machine learning to understand mutations. Bulletin of the American Physical Society 65, G20.08 (2020). 